\title{
Possible clinical implication of eosinophil extracellular traps in eosinophilic otitis media
}

Nobuo Ohta ${ }^{1,2 *}$, Shigeharu Ueki ${ }^{3}$, Makoto Hirokawa ${ }^{3}$, Sachiko Tomioka-Matsutani ${ }^{1}$, Tasuku Kawano ${ }^{4}$, Tomomitsu Miyasaka ${ }^{4}$, Tomoko Takahashi $^{4}$, Yutaka Nakamura ${ }^{5}$, Isao Ohno ${ }^{5}$ and Shigeharu Fujieda ${ }^{6}$

${ }^{1}$ Division of Otolaryngology, Tohoku Medical and Pharmaceutical University, Sendai, Japan

${ }^{2}$ Yamagata City Hospital Saiseikan, Yamagata, Japan

${ }^{3}$ Department of General Internal Medicine and Clinical Laboratory Medicine, Akita University Graduate School of Medicine, Akita, Japan

${ }^{4}$ Division of Pathophysiology, Department of Pharmaceutical Sciences, Faculty of Pharmaceutical Sciences, Tohoku Medical and Pharmaceutical University, Japan

${ }^{5}$ Center for Medical Education, Faculty of Medicine, Tohoku Medical and Pharmaceutical University, Japan

${ }^{6}$ Division of Otorhinolaryngology-Head \& Neck Surgery, Department of Sensory and Locomotor Medicine, Faculty of Medical Science, University of Fukui, Fukui, Japan

\begin{abstract}
Objectives: Eosinophilic otitis media (EOM) is an intractable middle ear disease characterized by a massive accumulation of eosinophils in middle ear effusions and mucosa. Eosinophil extracellular trap cell death (EETosis) is a newly established form of programmed cell death. To clarify the role of EETosis in EOM, we investigated middle ear effusions and clinical characteristics of patients with EOM.
\end{abstract}

Study design and setting: Case series with planned data collection.

Subjects and methods: The patient group consisted of 17 patients with EOM and 21 patients with chronic otitis media (COM). Eosinophilic extracellular traps (EETs) in middle ear effusions were microscopically evaluated.

Results: All patients with EOM had comorbid chronic rhinosinusitis with nasal polyps and bronchial asthma. The number of blood eosinophils, the level of IgE, and the density of eosinophils in effusions differed significantly between EOM and COM patients. Abundant EETs in highly viscous ear effusion were observed in all patients with EOM. Three patients with EOM had sensorineural hearing loss and the others had conductive and mixed hearing loss.

Conclusion: EETs were observed in the middle ear effusions of patients with EOM. These findings allow us to speculate that EETosis can increase the viscosity of middle ear effusions and thus be a potential therapeutic target.

\section{Introduction}

Eosinophilic otitis media (EOM) is a newly recognized intractable middle ear disease that is characterized by the strong infiltration of eosinophils in highly viscous middle ear effusions and in the middle ear mucosa [1,2]. Unlike conventional chronic otitis media (COM), the granulation tissue and highly viscous middle ear effusion of EOM are generally resistant to all therapies except for steroid treatment, and its aetiology is still ambiguous.

Eosinophil extracellular trap cell death (EETosis) is a novel form of programmed cell death [3]. In contrast to well-known cell death types such as apoptosis and necrosis, EETosis is characterized by the striking final morphology: release of web-like chromatin structures through breakdown of nuclear and plasma membranes [3,4]. We recently reported that EETosis is evident in association with diverse eosinophilassociated diseases. ${ }^{3), 4)}$ However, there are few reports of EETosis in EOM $[5,6]$. To clarify this point, we investigated the presence of EETosis in middle ear effusions of patients with EOM.

\section{Subjects and methods}

\section{Subjects}

Thirty-eight patients with EOM or COM were referred to the Department of Otolaryngology, Yamagata City Hospital Saiseikan, and the Tohoku Medical and Pharmaceutical University between February 2015 and March 2019. EOM was diagnosed according to previous reports as follows. Major criterion: EOM with effusion or COM with eosinophil-dominant effusion. Minor criteria (two or more): (1) Highly viscous middle ear effusion. (2) Resistance to conventional treatment for otitis media. (3) Association with bronchial asthma. (4) Association with nasal polyposis [1]. The Japanese Epidemiological Survey of Refractory Eosinophilic Chronic Rhinosinusitis score was used for evaluation of chronic rhinosinusitis with nasal polyps (CRSwNP) $[7,8]$. Audiometry was performed by experienced audiologists using a puretone audiometer (AA-76; Rion Co., Kokubunji, Japan) in a silent cabin. The pure-tone averages were evaluated against pure-tone audiograms using the American Academy of Otolaryngology Head and Neck Surgery's Committee on Hearing and Equilibrium guidelines. Pure-

${ }^{\star}$ Correspondence to: Nobuo Ohta, Division of Otolaryngology, Tohoku Medical and Pharmaceutical University, 1-12-1 Fukumuro Miyaginoku, Sendai, Japan, Tel: +81-22-259-1221, Fax: +81-22-259-1232; E-mail: noohta@hosp.tohoku-mpu.ac.jp

Key words: eosinophilic otitis media, eosinophil, extracellular trap cell death (EETosis), chronic otitis media, extracellular traps

Received: August 01, 2019; Accepted: August 16, 2019; Published: August 19 2019 
tone thresholds of 500,1000, 2000, 4000, and $8000 \mathrm{~Hz}$ were used for calculating mean hearing thresholds.

\section{Measurement of serum IgE levels}

Serum IgE concentrations in all patients were measured with a Behring nephelometer (Dade Behring, Deerfield, IL, USA) using antibodies against IgE.

\section{Detection of EETosis}

The middle ear effusion was fixed in $10 \%$ formalin and embedded in paraffin. For histone H1 staining, deparaffinized sample slides were incubated with $3 \%$ bovine serum albumin and $1 \%$ saponin in PBS for $30 \mathrm{~min}$ [3]. The slides were then incubated with primary mouse anti-human nuclear histone $\mathrm{H} 1$ monoclonal antibody (AE-4, $2 \mu \mathrm{g} /$ $\mathrm{mL}$, Abcam, $3 \mathrm{~h}$, at room temperature [RT]). Alexa-488-conjugated antibody (goat anti-mouse IgG; Invitrogen, Carlsbad, CA, USA; 1:300, $30 \mathrm{~min}$, at RT) was used as secondary antibody. Control antibodies and Hoechst 33342 stain (Invitrogen) were used in each experiment. To study the 3D structure of DNA traps, we stained each fixed specimen with SYTOX Green nucleic acid stain (Invitrogen), took images with a Carl Zeiss LSM510 confocal microscope, and created Z-stack images in Zeiss LSM software. Histone H1 was used as a maker of nucleusderived DNA to distinguish it from mitochondrial DNA.

\section{Assessment of slides}

Immunostained sections were assessed under an Olympus microscope with an eyepiece reticle at $200 \times$ magnification [3]. Cell counts are expressed as means per high-power field $\left(0.202 \mathrm{~mm}^{2}\right)$. At least two sections were immunostained, and more than five areas were evaluated via the graticule. Results are expressed as number of positive cells per field as follows: -, negative;,$+<10$ cells in each high-power field $(\times 400)$;,$++ 10-20$ cells;,$+++>20$ cells.

\section{Statistical analysis}

All data were analyzed by Student's $t$-test, Wilcoxon's rank-sum test, or the Mann-Whitney $U$-test in SPSS v. 20 software (IBM, Chicago, IL, USA). Differences with a corrected $P$-value of $<0.05$ were considered significant. Data are presented as mean $\pm \mathrm{SD}$.

The study was approved by the Tohoku Medical and Pharmaceutical University Hospital Institutional Review Board (2016-2-019).

\section{Results}

Clinical features of the patients with EOM or COM are summarized in table 1. All patients with EOM had comorbid CRSwNP and bronchial asthma (BA). There were significant differences in serum IgE levels and in the number of eosinophils between EOM and COM (Table 1). All patients with EOM had hearing impairment, perforated tympanic membrane, highly viscous middle ear effusion, and granulation in the middle ear cavity, and all patients with COM had mucopurulent otorrhea (Table 2). All patients with EOM had massive infiltration of eosinophils with cytolytic degranulation and abundant EETs in the highly viscous ear effusion. Recurrence was more frequent in EOM than in COM. Three patients with EOM had sensorineural healing loss and were successfully treated with systemic corticosteroids. They are presented as follows.

Case 1: From 2010, a 25-year-old Japanese man was treated by an ENT clinic for right hearing loss and right middle ear effusion with antibiotics for COM and CRSwNP. In March 2015 he was referred to us. We found bilateral nasal polyps and performed endoscopic sinus
Table 1. Clinical characteristics of patients with EOM or COM

\begin{tabular}{|l|l|l|l|}
\hline Characteristic & EOM & COM & Significance \\
\hline Sex (male, female), $n$ & 8,9 & 10,11 & ns \\
\hline Age at onset, years, median (range) & $37(27-69)$ & $28.9(11-79)$ & $\mathrm{ns}$ \\
\hline Age at enrollment, years, median (range) & $42.1(27-74)$ & $31.6(11-71)$ & $\mathrm{ns}$ \\
\hline Hearing levels, median (range) dB & $39.4(30-45)$ & $42.1(32-68)$ & $\mathrm{ns}$ \\
\hline Comorbidity of nasal polyps, $n(\%)$ & $17(100)$ & $1(4.8)$ & $P<0.05$ \\
\hline Comorbidity of allergic rhinitis, $n(\%)$ & $12(70.2)$ & $4(19)$ & $P<0.05$ \\
\hline Comorbidity of bronchial asthma, $n(\%)$ & $17(100)$ & 0 & $P<0.05$ \\
\hline IgE (IU/mL), mean \pm SD & $428.1 \pm 328.5$ & $151.8 \pm 132.5$ & $P<0.05$ \\
\hline Eosinophils, cells/mL, mean \pm SD & $579.2 \pm 299.8$ & $231.8 \pm 167.5$ & $P<0.05$ \\
\hline Neutrophil, cells/mL, mean \pm SD & $3891 \pm 2546$ & $3972 \pm 2816$ & $\mathrm{~ns}$ \\
\hline Eosinophilic infiltration count in nasal polyp, $n$ & $156.3 \pm 149.2$ & n.d. & ns \\
\hline Recurrence, $n$ (\%) & $16(94 \%)$ & $4(11.2)$ & $P<0.05$ \\
\hline JESREC score & $15.1 \pm 2.6$ & n.d. & ns \\
\hline
\end{tabular}

AR: Allergic Rhinitis; Chronic Rinosinusitis with Nasal Polyp; CRSwNP ICS: Inhaled Corticosteroids; IgE: Immunoglobulin E; nd: Not Done; ns: Not Significant; JESREC: Japanese Epidemiological Survey of Refractory Eosinophilic Chronic Rhinosinusitis

Table 2. Pathological characteristics of ear effusion. ns: Not Significant; nd: Not Done.

\begin{tabular}{|l|l|l|l|}
\hline Occurrence, $\boldsymbol{n}(\%)$ & EOM & COM & Significance \\
\hline Perforation of tympanic membrane & $17(100)$ & $21(100)$ & ns \\
\hline Highly viscous otorrhea & $17(100)$ & 0 & $P<0.05$ \\
\hline Mucopurulent otorrhea & $2(11.8)$ & $21(100)$ & $P<0.05$ \\
\hline Eosinophil-rich middle ear effusion & $17(100)$ & 0 & $P<0.05$ \\
\hline Neutrophil-rich middle ear effusion & $2(11.8)$ & $21(100)$ & $P<0.05$ \\
\hline EETosis & $17(100)$ & 0 & $P<0.05$ \\
\hline
\end{tabular}

surgery (ESS) (Figure 1a). After surgery, the patient still experienced hearing loss and effusion. We observed a central perforated tympanic membrane with highly viscous middle ear effusion and granulation (Figure 1b). Computed tomography (CT) scans of the right middle ear revealed a soft tissue density mass in the right upper and middle tympanic cavity (Figure 1c). An audiogram demonstrated mixed hearing loss of 15 to $25 \mathrm{~dB}$ associated with an air-bone gap and sensorineural hearing loss at 1000 and $2000 \mathrm{~Hz}$ (Figure 1d). Pathological examination revealed massive infiltration of eosinophils in the highly viscous middle ear effusion (Figure 1e). EETs were detected in the effusion (Figure 1f). With daily treatment with prednisolone $30 \mathrm{mg}$, the effusion and granulation declined rapidly. After tapering of prednisolone, monthly anti-IgE therapy for comorbid BA has administered. The recurrence of EOM was not observed to date.

Case 2: In 2016, this woman, who is now 48 years old, was treated by our department of internal medicine for a complaint of chest pain. The diagnosis was eosinophilic pneumonitis. In 2017, she consulted us because hyposmia had appeared. CT revealed nasal polyps and a soft tissue density mass in both ethmoid sinuses. The diagnosis was CRSwNP. In the same year, she underwent ESS. In 2018, she lost hearing three times, so she was admitted to our department. On admission she had a hyperemic external auditory meatus and a perforated tympanic membrane (Figure 2a). CT of the left middle ear revealed a soft tissue density mass in the left upper and middle tympanic cavity (Figure 2b). She had slight sensorineural hearing loss at 2000 and $4000 \mathrm{~Hz}$ (Figure 2c). Pathological examination revealed massive infiltration of eosinophils in a highly viscous middle ear effusion (Figure 2d). EETs were detected in the effusion (Figure 2e). With daily treatment with prednisolone $30 \mathrm{mg}$, the effusion and granulation declined rapidly.

Case 3: In August 2015, this man, who is now 34 years old, complained of abnormal smell perception. In February 2016, he was treated by an otolaryngologist, but his smell perception did not improve. From March onward he was treated with prednisolone $15 \mathrm{mg}$ 
a)
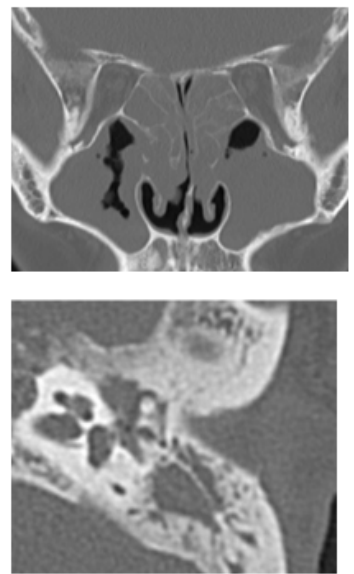

c) b)
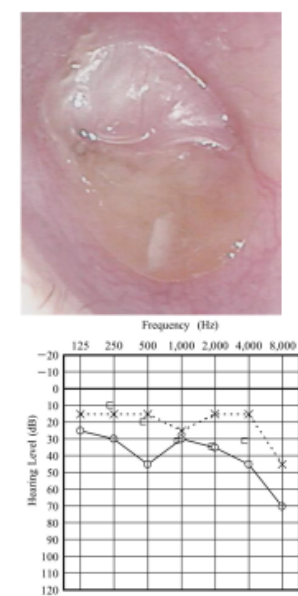

d)

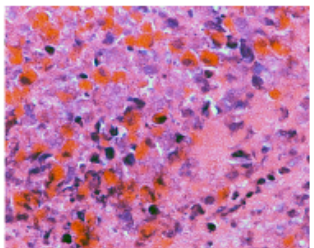

e)

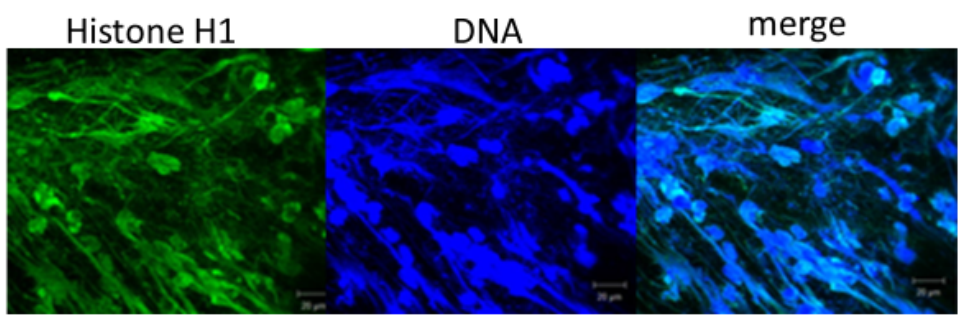

f)

Figure 1. EOM affecting a 25-year-old Japanese man. (a) Semicoronal section of CT revealed nasal polyps and soft tissue density mass in both ethmoid sinuses. (b) Perforation of tympanic membrane associated with granulation tissue and highly viscous discharge. (c) Axial CT scan shows soft tissue density mass in the upper and middle tympanic cavity. (d) Audiogram shows right conductive hearing loss of 25-45 dB in air-bone gap and sensorineural hearing loss at 1000 and $2000 \mathrm{~Hz}$. (e) Pathological examination revealed massive eosinophil infiltration and cytolysis of middle ear mucosa (H\&E, $\times 200)$. (f) Smear of highly viscous middle ear effusion stained for linker-histone H1 (green) and DNA (Hoechst 33342 , blue) and analyzed by laser scanning confocal microscope. Scale bar, $20 \mu \mathrm{m}$. Images show net-like formation of histone and DNA in middle ear effusion. This finding indicates EETs.

a)

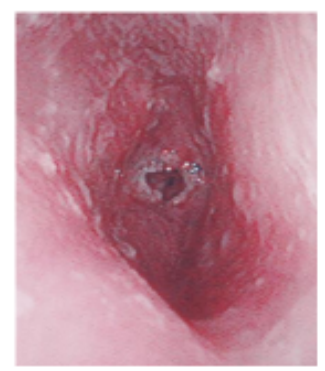

Freyeacy $(\mathrm{H} 2)$

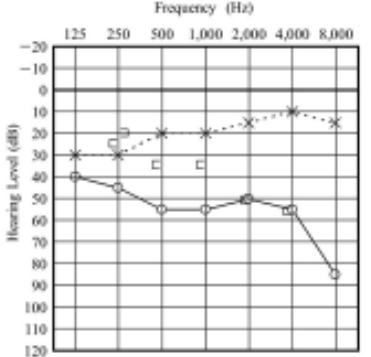

c) b)
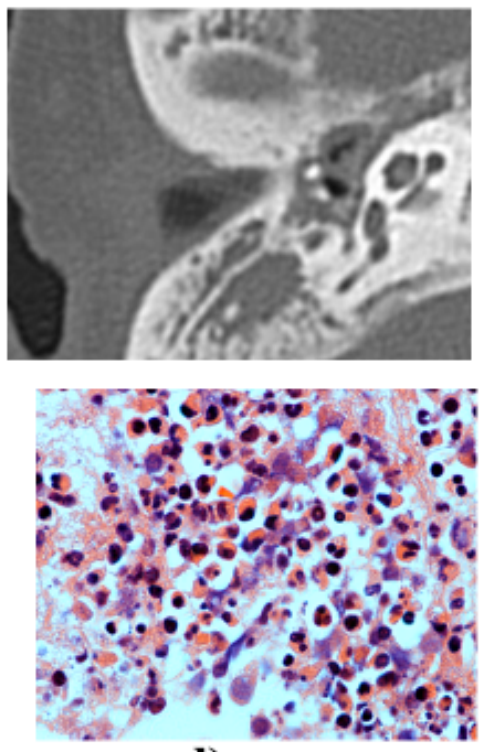

d)

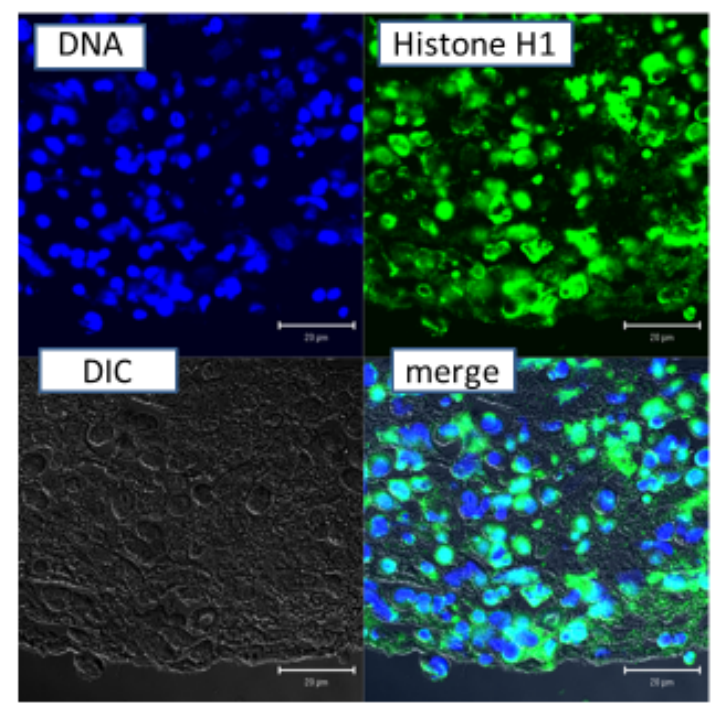

e)

Figure 2. EOM affecting a 48-year-old Japanese woman. (a) Hyperemic external auditory meatus and tympanic membrane with perforation. (b) CT scan shows soft tissue density mass in the meso-tympanic cavity. (c) Audiogram shows right sensorineural hearing loss at 2000 and $4000 \mathrm{~Hz}$. (d) Pathological examination revealed massive eosinophilic infiltration of middle ear mucosa (H\&E, $\times 200)$. (e) Paraffin section of highly viscous middle ear effusion stained for linker-histone H1 (green) and DNA (Hoechst 33342 , blue) and analyzed by laser scanning confocal microscope. Scale bar, $20 \mu \mathrm{m}$. Histone and DNA in the middle ear granulation. This finding indicates EETs.

daily. His symptoms improved temporarily but recurred on cessation of treatment. He also developed nasal obstruction. On resumption of steroid therapy his symptoms declined. In April 2016, he was referred to us. We found a left highly viscous middle ear effusion and conductive hearing loss. Pathological examination revealed massive infiltration of eosinophils in highly viscous middle ear effusion. EETs were detected in the effusion. With daily treatment with prednisolone, the effusion and granulation declined rapidly. The patient's symptoms did not recur following treatment.

\section{Discussion}

EOM is a newly recognized intractable middle ear disease that is characterized by the accumulation of eosinophils in highly viscous middle ear effusion and in the middle ear mucosa [1]. Although its prevalence is not well known, it is more prominent in patients aged 50-60 years and in women. Among all patients with EOM, 75\% of cases are associated with CRSwNP and BA [1]. Notably, BA is the disease most frequently (89.9\%) associated with EOM [1], and the aetiologies 
of asthma, ECRS, and EOM are thus considered to be closely related to each other. All of our patients with EOM had comorbid CRSwNP and BA.

The control of highly viscous middle ear effusion and hearing loss is very important for ENT doctors as a strategy for the treatment of EOM [1-3]. The instillation of triamcinolone acetonide or salineheparin solution into the tympanic cavity has been used as local treatment $[1,9,10]$. Although the use of systemic corticosteroid is the first-line treatment and is generally effective, recurrent or refractory cases are common in EOM [11]. Patients with EOM have a high risk of conductive hearing loss in the early stage, and half of all patients develop an increased bone-conduction threshold, which leads to deafness in $6 \%$ of cases $[1,10,12]$. Here, 3 of 17 patients with EOM had sensorineural healing loss and were successfully treated with systemic corticosteroids. However, in cases with bilateral deafness, cochlear implantation should be considered [13-15].

EETosis is a newly established form of programmed cell death [35]. We detected EETs in highly viscous middle ear effusions obtained from all patients with EOM. Purified DNA is very sticky and highly viscous, just like the middle ear effusion from these patients [5]. These results indicate that EETosis-derived eosinophil DNA traps are major components of eosinophil-rich, highly viscous middle ear effusions in EOM and contribute to their viscosity.

The mechanism underlying sensory hearing loss in EOM is still a mystery. However, not only the middle ear but also the inner ear, including the cochlea, scala tympani of the organ of Corti, and capillaries of the stria vascularis, were infiltrated with eosinophils to variable degrees in an animal model of EOM [13-16]. Taken together, these results suggest that eosinophilic inflammation in the middle ear could influence inner ear function directly [13-16]. Interestingly, we found that three EOM patients with sensorineural hearing loss had a stronger expression of EETosis than patients with conductive hearing loss. These findings allow us to speculate that EETosis can be used as an additional indicator of disease activity and as a biomarker for potential sensorineural hearing loss.

EETs are major extracellular structural components scaffolding the eosinophilic secretions and increasing their viscosity in EOM. Regulating excess EETosis could be a novel target for treating EOM. Therefore, further studies are required to more fully characterize EOM in more cases and to clarify the long-term outcomes.

\section{Conclusion}

In EOM, EETs in eosinophil-rich middle ear effusions was observed. These findings allow us to speculate that EETosis increases the viscosity of middle ear effusion and could be used as an additional indicator of disease activity and as a biomarker for potential sensorineural hearing loss.

\section{Acknowledgments}

This work was supported by a Grant-in-Aid for Scientific Research (C), grant number 17K11363, from the Japan Society for the Promotion of Science and the Ministry of Health, Labor, and Welfare of Japan. We express our sincere thanks to Mrs. Yuko Ohta, Uyo Gakuen College, and Dr. Toshinori Kubota, Department of Otolaryngology Head and Neck Surgery, Yamagata University Faculty of Medicine, for their editorial assistance.

\section{References}

1. Iino Y, Tomioka-Matsutani S, Matsubara A, Nakagawa T, Nonaka M (2011) Diagnostic criteria of eosinophilic otitis media, a newly recognized middle ear disease. Auris Nasus Larynx 38: 456-461. [Crossref]

2. Ohta N, Ishida A, Kurakami K, Suzuki Y, Kakehata S, et al. (2014) Expressions and roles of periostin in otolaryngological diseases. Allergol Int 63: 171-180. [Crossref]

3. Ueki S, Konno Y, Takeda M, Moritoki Y, Hirokawa M, et al. (2016) Eosinophil extracellular trap cell death-derived DNA traps: Their presence in secretions and functional attributes. J Allergy Clin Immunol 137: 258-267. [Crossref]

4. Ueki S, Ohta N, Takeda M, Konno Y, Hirokawa M (2017) Eosinophilic Otitis Media The Aftermath of Eosinophil Extracellular Trap Cell Death. Curr Allergy Asthma Rep 17: 33. [Crossref]

5. Ohta N, Ueki S, Konno Y, Hirokawa M, Kubota T, et al. (2018) ETosis-derived DNA trap production in middle ear effusion is a common feature of eosinophilic otitis media. Allrgol Int 67: 414-416. [Crossref]

6. Ueki S, Konno Y, Takeda M, Moritoki Y, Hirokawa M, et al. (2016) Eosinophil extracellular trap cell death-derived DNA traps: Their presence in secretions and functional attributes. J Allergy Clin Immunol 137: 258-267. [Crossref]

7. Tokunaga T, Sakashita M, Haruna T, Asaka D, Takeno S, et al. (2015) Novel scoring system and algorithm for classifying chronic rhinosinusitis: the JESREC Study. Allergy 70: 995-1003. [Crossref]

8. Fujieda S, Imoto Y, Kato Y, Ninomiya T, Tokunaga T, et al. (2019) Eosinophilic chronic rhinosinusitis. Allergol Int: S1323-8930(19)30103-0.

9. Seo Y, Nonaka M, Tagaya E, Tamaoki J, Yoshihara T (2015) Eosinophilic otitis media is associated with asthma severity and smoking history. ORL J Otorhinolaryngol Relat Spec 77: 1-9. [Crossref]

10. Iino Y (2010) Role of IgE in eosinophilic otitis media. Allergol Int 59: 233-238. [Crossref]

11. Ishida A, Ohta N, Suzuki Y, Kakehata S, Okubo K, et al. (2012) Expression of pendrin and periostin in allergic rhinitis and chronic rhinosinusitis. Allergol Int 61: 589-595. [Crossref]

12. Kanemitsu Y, Matsumoto H, Izuhara K, Tohda Y, Kita H, et al. (2013) Increased periostin associates with greater airflow limitation in patients receiving inhaled corticosteroids. J Allergy Clin Immunol 132: 305-312. [Crossref]

13. Nishizawa H, Matsubara A, Nakagawa T, Ohta N, Izuhara K, et al. (2012) The role of periostin in eosinophilic otitis media. Acta Otolaryngol 132: 838-844. [Crossref]

14. Cunha AA, Porto BN, Nuñez NK, Souza RG, Vargas MH, et al. (2014) Extracellular DNA traps in bronchoalveolar fluid from a murine eosinophilic pulmonary response. Allergy 69: 1696-700. [Crossref]

15. Sugimoto H, Hatano M, Noda M, Hasegawa H, Yoshizaki T (2016) Cochlear implantation in deaf patients with eosinophilic otitis media using subtotal petrosectomy and mastoid obliteration. Eur Arch Otorhinolaryngol 274: 1173-1177. [Crossref]

16. Matsubara A, Nishizawa H, Kurose A, Nakagawa T, Takahata J, et al. (2014) An experimental study of inner ear injury in an animal model of eosinophilic otitis media. Acta Otolaryngol 134: 227-232. [Crossref]

Copyright: (C2019 Ohta N. This is an open-access article distributed under the terms of the Creative Commons Attribution License, which permits unrestricted use, distribution, and reproduction in any medium, provided the original author and source are credited. 